\title{
Transient Response of Single-Domain Y-Ba-Cu-O Rings to Pulsed Magnetic Fields
}

\author{
T. R. Askew, J. M. Weber, Y. S. Cha, H. Claus, B. W. Veal
}

\begin{abstract}
Shielding current limits and magnetic diffusion characteristics have been measured at $77 \mathrm{~K}$ for a set of YBCO single-domain rings. These were fabricated from melt-textured cylindrical YBCO monoliths that were densified to nearly $100 \%$, and then oriented from a single seed. The rings were surrounded by a drive coil that can, under pulse conditions, achieve applied magnetic fields in excess of $1 \mathrm{~T}$ and induce currents in excess of $50 \mathrm{kA}$. Simultaneous magnetic characterization with a Rogowski coil and Hall probe was used to determine the induced current in the sample and the magnetic field in the center of the sample. Magnetic fields trapped in the samples were mapped with a scanning Hall probe. When compared with similar measurements on multidomain c-axisoriented YBCO rings, the flux penetration is faster and more uniform around the circumference of the ring. The observed critical current density, $\approx 15,000 \mathrm{~A} / \mathrm{cm}^{2}$ at $77 \mathrm{~K}$, is suitable for application in penetration-type fault current limiters. Separate measurements of the trapped magnetic field and critical current density in the rings are compared with results obtained by analysis of magnetic diffusion characteristics.
\end{abstract}

Index Terms-Single Domain YBCO Rings, Magnetic Diffusion, Magnetic Shielding, Pulsed Current Testing, Scanning Hall Probe

\section{INTRODUCTION}

$\mathrm{P}$ rogress in $\mathrm{YBa}_{2} \mathrm{Cu}_{3} \mathrm{O}_{7}$ (YBCO) top-seeded melt-growth technology has enabled the fabrication of single-domain cylindrical monoliths with diameters of several centimeters and critical current densities $\left(J_{c}\right)$ at $77 \mathrm{~K}$ that are attractive for many applications [1]. This study explores the possible use of this type of material in inductive fault current limiters (FCLs) and compares the response to pulsed magnetic fields at $77 \mathrm{~K}$ with that previously observed for $\mathrm{Bi}_{2} \mathrm{Sr}_{2} \mathrm{CaCu}_{2} \mathrm{O}_{\mathrm{x}}$ (BSCCO) tubes [2] and $\mathbf{c}$-axis oriented multidomain $\mathrm{YBCO}$ rings [3]. The samples are part of a population recently used to explore successful "welding" of monoliths together [4] and

Manuscript received August 5, 2002. This work was supported by the U.S. Department of Energy, Energy Efficiency and Renewable Energy, as part of a program to develop electric power technology, under Contract W31-109-Eng-38.

T. R. Askew and J. M. Weber are with the Physics Department of Kalamazoo College, Kalamazoo, MI (phone: 616-337-7097; fax: 616-3377101; e-mail: askew@kzoo.edu), and with Energy Technology Division, Argonne National Laboratory.

Y. S. Cha is with the Energy Technology Division, Argonne National Laboratory, Argonne, IL USA (phone: 630-252-5899; fax: 630-252-5568; email: yscha@anl.gov).

H. Claus and B. W. Veal are with the Materials Science Division, Argonne National Laboratory, Argonne, IL USA (e-mail: claus@anl.gov/ veal@anl.gov). the effects of the resultant low-angle grain boundaries on the $J_{c}$ [5]. Applications that require parts larger than a few centimeters thus become possible if the limits on $J_{c}$ imposed by the welding process (about $10^{4} \mathrm{~A} / \mathrm{cm}^{2}$ at $77 \mathrm{~K}$ ) can be tolerated.

The essential parts of a penetration-type inductive FCL are an iron core or yoke inside a superconducting ring or tube and a copper drive coil that is inductively coupled to both the superconductor and the iron core. When the FCL is used under normal operating conditions, the impedance is low because the magnetic flux from the drive coil is shielded by the superconductor and only weakly coupled into the iron core. Under fault conditions, the large current in the copper drive coil produces a magnetic field that exceeds the shielding capability of the superconductor tube. This large field then penetrates into the iron core, couples into the yoke, and causes the impedance of the drive coil to rise sharply. Under voltagelimited conditions, the increase in impedance causes the current in the drive coil to drop, thereby achieving a useful current-limiting effect that can be applied in power distribution systems. Depending on magnetic and electrical circuit conditions, the change in impedance might be primarily resistive or inductive [6].

Proper understanding of the thermal and electromagnetic details of the penetration process requires that they be investigated under cylindrically symmetric conditions. Achievement of optimal device performance may also require symmetric penetration. Uniform behavior among a group of samples is important if multiple rings are used in the same FCL, or if a single ring is welded together from multiple pieces. Nonuniformities in flux pinning in the YBCO rings were explored by cooling the rings in an applied magnetic field and then mapping the resultant trapped-field distribution under static conditions at $77 \mathrm{~K}$. These static, trapped-field measurements correctly predict the variation from sample to sample in ring critical currents $\left(I_{c}\right)$ observed under pulsed-field conditions. Nonuniformities in the flux pinning of individual samples also correlate with the observed dynamic response, with samples that show good cylindrical symmetry in their flux pinning producing the sharpest magnetic response at the instant of penetration.

\section{EXPERIMENTAL DETAILS}

Eight rings were prepared from cylindrical monoliths that were $28 \mathrm{~mm}$ in diameter. These were sliced with a diamond 
saw into disks that were from 4 to $8 \mathrm{~mm}$ thick. Then, the centers were cut out with an 11-mm-dia. brass core drill using diamond grit. The monolith preparation process was designed to minimize the presence of voids and second-phase $\left(\mathrm{Y}_{2} \mathrm{Ba}_{1} \mathrm{Cu}_{1} \mathrm{O}_{\mathrm{x}}\right)$ material and to maximize the uniformity and completion of the final oxygenation. Specific details are given elsewhere [5]. The resultant material is free of voids and nearly $100 \%$ dense, with a single phase. Seven of the rings exhibited critical current values $\left(I_{c}\right)$ at $77 \mathrm{~K}$ between 7 and $18 \mathrm{kA}$, while one sample exhibited an $I_{c}$ of only $1.3 \mathrm{kA}$. This last sample had a visible radial crack and appears in the following analysis as Sample C, showing the influence of the crack on the various measurements. Despite their common origin and similar appearance, the remaining samples exhibited $J_{c}$ values between 12 and $31 \mathrm{kA} / \mathrm{cm}^{2}$, illustrating the need to analyze multiple samples in this type of investigation.

Fig. 1 illustrates the origin of some of the variations in sample properties. The seed in a top-down view of the monolith, looking down the caxis, is shown in Fig. 1a. Oriented growth proceeds outward, in the a-b direction, forming growth planes. Later measurements show that flux pinning is often stronger in regions where the planes intersect, causing secondary current distributions around the pinning centers, shown in Fig. 1b, that add to the primary current circulating around the ring. Cracks, as in Sample $\mathrm{C}$, cause current crowding and a reduced $I_{c}$ for the structure. Because the material is designed to have a uniform, modest flux pinning, it does not exhibit the $J_{c}$ values of $100 \mathrm{kA} / \mathrm{cm}^{2}$ or more seen at $77 \mathrm{~K}$ when various defects or second-phase morphologies are manipulated to produce additional pinning [7]. The results that follow will emphasize Sample A, a ring with modest, but very uniform pinning, Sample B, a ring with stronger, but less uniform pinning, and Sample C, already mentioned.
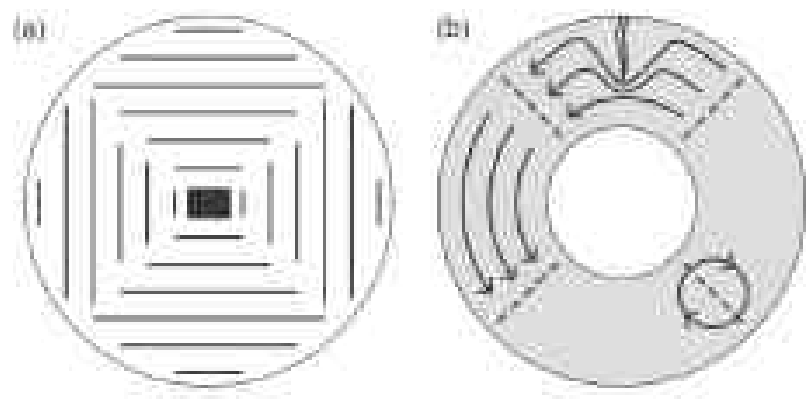

Fig. 1. (a) View of top-seeded cylindrical monolith. (b) Schematic of resultant YBCO ring with pinning centers (---), primary and secondary circulating currents, and current crowding around a crack.

The choice of test configuration is motivated by the geometry of an inductive FCL, and is shown in cross section, to scale, in Fig. 2. The drive coil consists of 75 turns of AWG 18 copper wire, and is driven by a capacitive-discharge pulsed current supply that is described elsewhere [8]. The integrated signal from a Rogowski coil is used in the conventional way $[9,10]$ to calculate the sum of the current flowing in the drive coil and the induced current flowing around the superconducting ring. The drive coil current is measured separately on a shunt, allowing the circulating ring current (primary current of Fig. 1b) to be obtained by subtraction. A Hall probe measures the vertical (c axis) component of the magnetic flux density. For trapped-field measurements, the sample is cooled to $77 \mathrm{~K}$ in an applied field of $0.3 \mathrm{~T}$, and then the field is removed. The Hall probe is then scanned over a plane $\approx 1 \mathrm{~mm}$ above the top surface of the sample to record the static distribution of the trapped field. For measurements under dynamic conditions, the Hall probe is inserted into the hole in the center, and held on the central axis in the medial plane. All measurements (Hall voltage, shunt voltage, Rogowski coil voltage) are performed with 16bit A/D conversion at time constants that are much shorter than any phenomena in the pulse characteristics or test system response. Because geometrical and equipment constraints prevent the simultaneous achievement of certain combinations of measurements, multiple runs under identical conditions were used to obtain entire data sets.

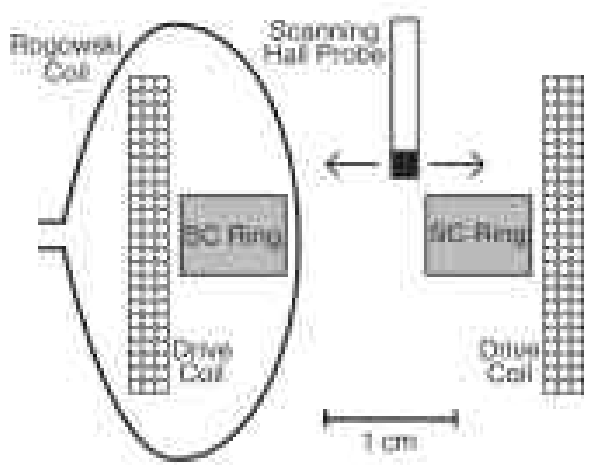

Fig. 2. Cross section of test configuration, drawn to scale.

\section{RESULTS ON INDIVIDUAL RINGS}

Fig. 3 illustrates the coil drive current $N I$ (in kA·turns), the induced ring current $I_{S}$ in Sample A (kA), and the magnetic flux density $B(\mathrm{~T})$ in the sample center, all as a function of time during a pulse that peaks at $3 \mathrm{~ms}$. The induced ring current is shown as less than zero because its direction is opposed to the NI. Under ideal conditions, these currents would be equal and opposite, but flux leakage in the experimental geometry causes a mismatch of $\approx 2.5: 1$. Fig. $3 \mathrm{a}$ shows the response of Sample A to a current pulse of 23 $\mathrm{kA} \cdot$ turns, a value less than that required for penetration. The magnetic flux density in the center of the sample climbs slowly as the applied field diffuses into the sample under flux creep conditions. Fig. $3 b$ shows the same information for a current pulse of $25 \mathrm{kA}$ turns, a value right on the threshold of penetration. Fig. 3c shows results for a pulse of $27 \mathrm{kA}$.turns, enough to cause a distinct penetration at $3 \mathrm{~ms}$. A dramatic jump in the rate of change of the magnetic field at the sample center $(\partial B / \partial t)$ can be observed at $3 \mathrm{~ms}$, as large portions of the sample switch from flux creep to flux flow. Heating of the ring, due to dissipation under flux flow, reduces the size of the peak $I_{S}$ from $\approx 9.5 \mathrm{kA}$ in Fig. $3 \mathrm{a}$ to $8 \mathrm{kA}$ in Fig. $3 \mathrm{c}$. The time interval between successive data points in these plots is $200 \mu \mathrm{s}$. 

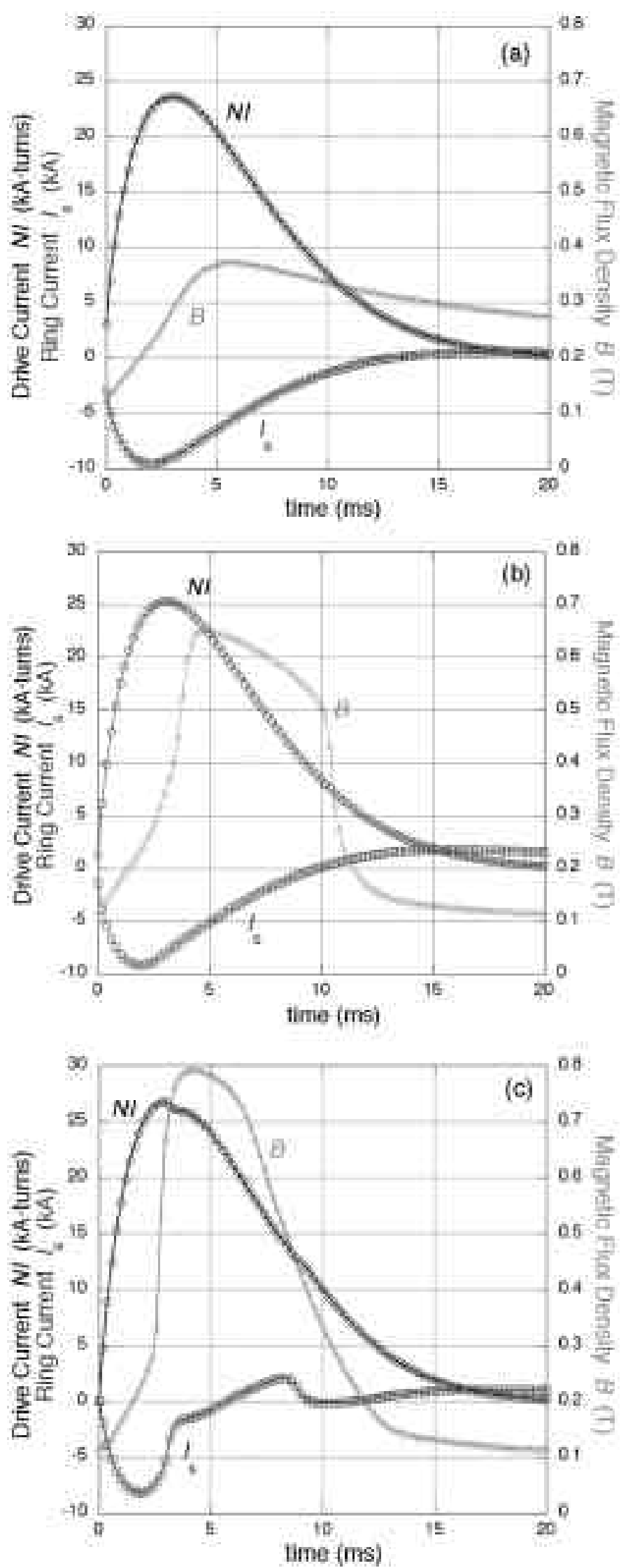

Fig. 3. Drive coil current (positive, in kA.turns), induced ring current (negative, in kA), and axial magnetic flux density for Sample A below penetration (a), at penetration (b), and beyond penetration (c).
Fig. 4 shows the trapped-field pattern for Sample A, after being cooled to $77 \mathrm{~K}$ in a $0.3-\mathrm{T}$ field. Four peaks of nearly equal height are observed, corresponding to the locations of stronger pinning shown in Fig. 1. The field at the point above the center of the sample is $0.11 \mathrm{~T}$.

Fig. 5 shows similar data to that of Figs. $3 \mathrm{c}$ and 4, but for Sample B, which exhibits much higher but much less uniform flux pinning than Sample A. The maximum $I_{S}$ is $18 \mathrm{kA}$, which corresponds to a $J_{c}$ of $31 \mathrm{kA} / \mathrm{cm}^{2}$. Other than larger currents and field values, the main difference between Samples $\mathrm{A}$ and $\mathrm{B}$ is that the latter does not show the sharp change in $\partial B / \partial t$ at penetration. This finding suggests that the $\mathrm{B}$ ring is not penetrated symmetrically, with the result that the observed field at the center is a complicated spatial average of what is happening at differing rates in various sectors of the ring. There is no obvious performance penalty or additional time delay associated with this. This pattern, of which A and B represent extreme cases, is followed by the seven samples that exhibit $J_{c}$ values of $15 \mathrm{kA} / \mathrm{cm}^{2}$ or more.

In contrast, Fig. 6 shows similar results for Sample C, which contained an observable crack and much lower values of $I_{S}$. The resultant penetration is extremely sharp, with a sudden jump in magnetic field overrunning the $50 \mathrm{kHz}$ bandwidth of the Hall probe measurement system and causing inductive "ringing." Sudden reduction of the circulating current in one sector of the ring produced a response that is quite different from that of nonsymmetric field penetration, and a transition from flux creep to flux flow throughout the entire ring, but not necessarily at the same time in each sector.

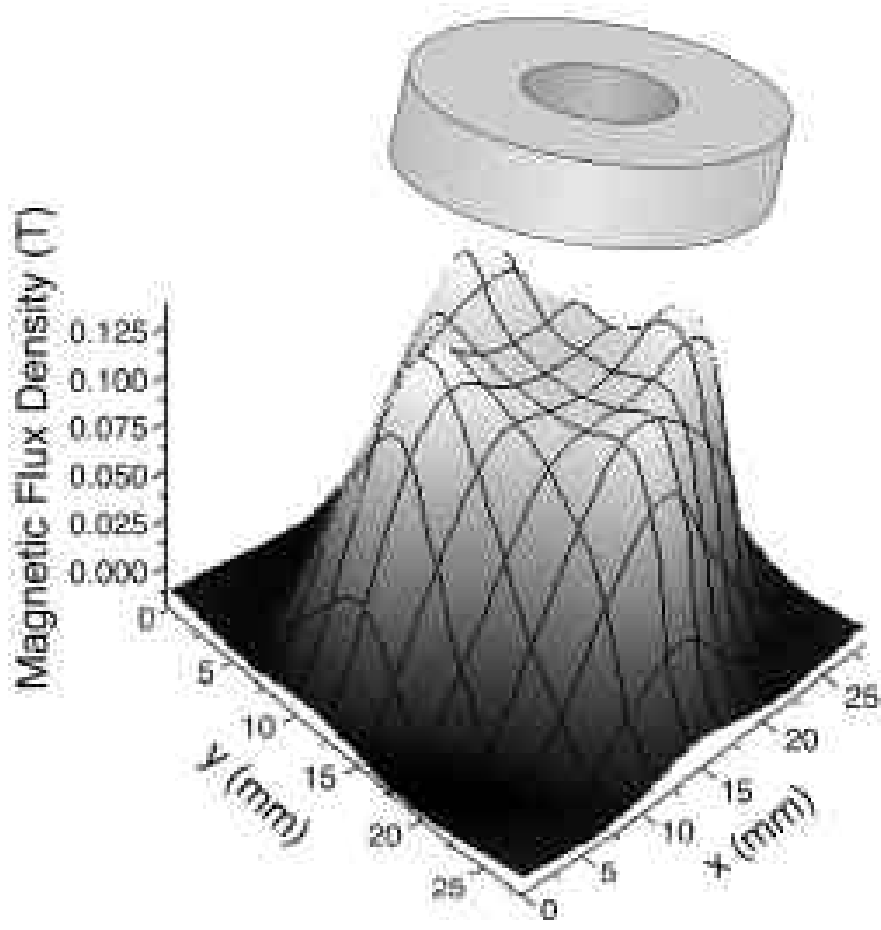

Fig. 4. Scanning Hall probe measurements of the trapped magnetic flux density in Sample A. 


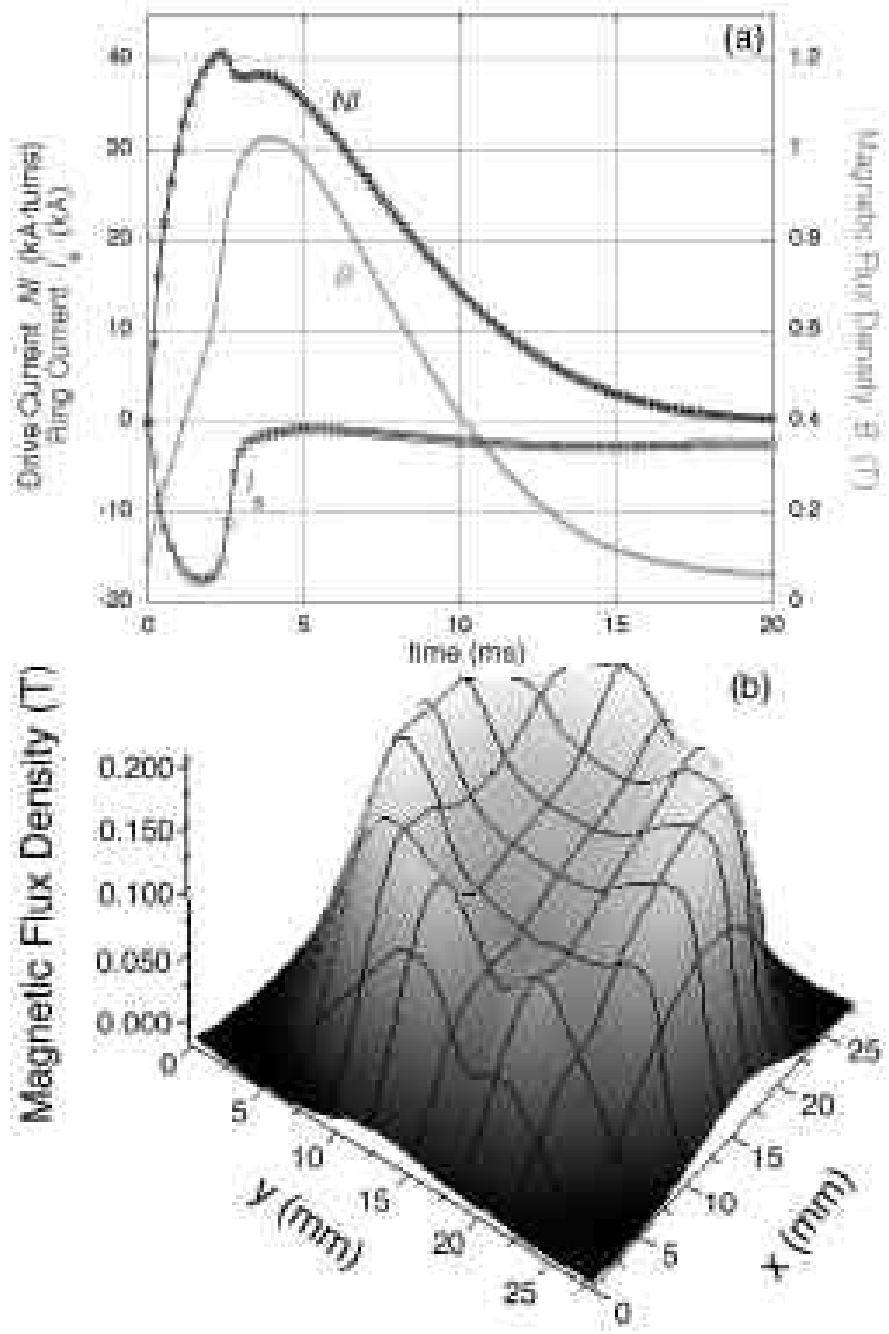

Fig. 5. (a) Drive coil current (positive, in kA·turns), induced ring current (negative, in kA), and axial magnetic flux density for Sample B beyond penetration. (b) Scanning Hall probe measurements of Sample B.

\section{DISCUSSION AND COMPARISON OF VARIOUS TYPES OF MEASUREMENTS}

It is known from other studies [4] that the magnitude of the trapped field in the center of the ring (actually measured above the center of the ring) is a good indicator of the size of the circulating primary critical current and only weakly influenced by secondary current distributions (see Fig. 1). Assuming that all of the trapped field at the center is due to primary current, and that all of that current flows at the critical current density (the simple Bean model), one can calculate the $J_{c}$ necessary to produce the measured trapped field. These $J_{c}$ values are shown in Fig. 7, with the measured trapped magnetic fields above the center shown on the left vertical axis, and the corresponding calculated $J_{c}$ values on the right vertical axis. The horizontal axis indicates $J_{c}$ values for the same samples that are calculated from the maximum $I_{S}$ induced under dynamic conditions, just before penetration, as in Fig. 3a. Data for the three rings discussed earlier, A, B and $\mathrm{C}$, are indicated.

The two measurements are correlated up to "dynamic" $J_{c}$
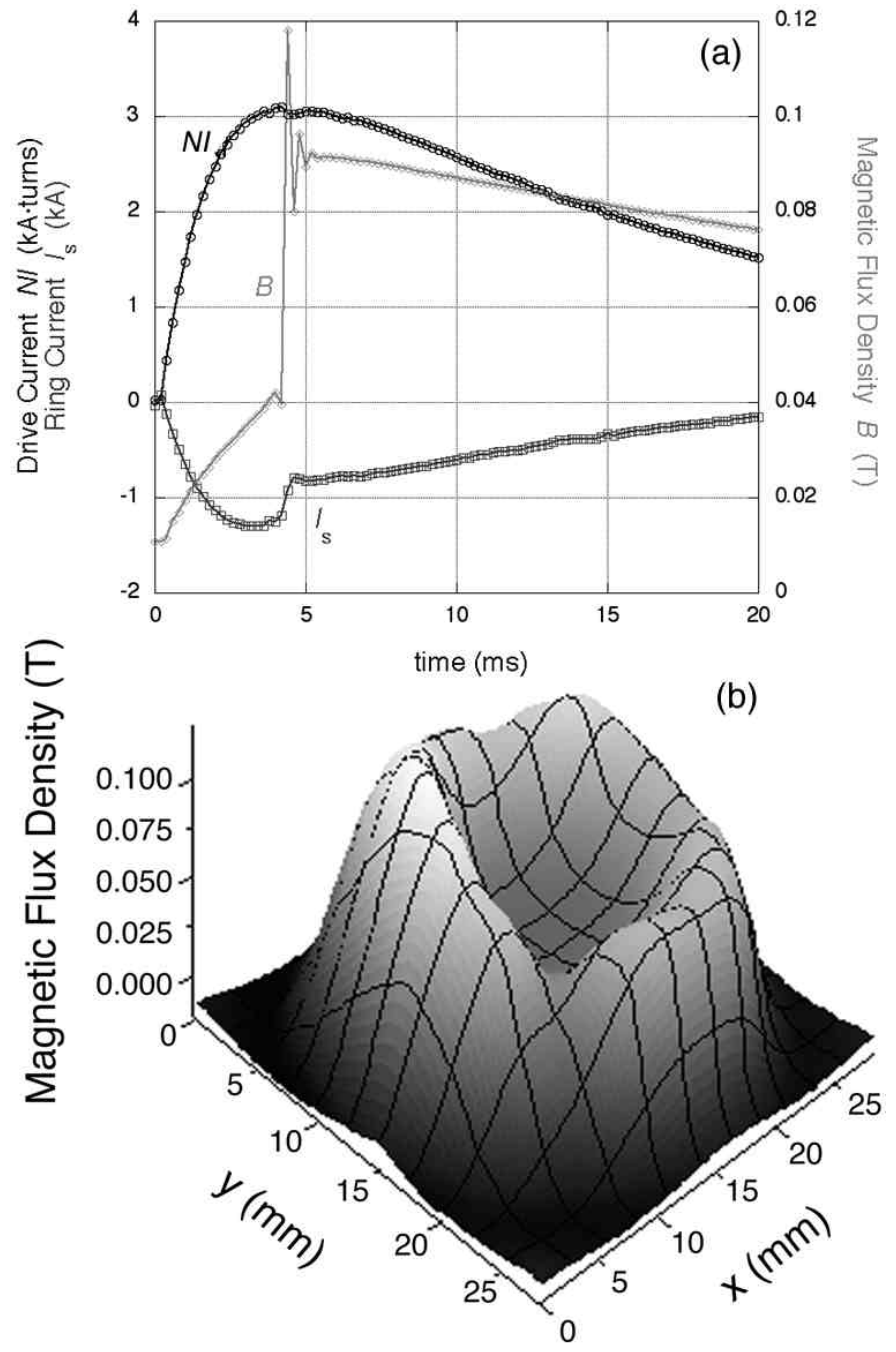

Fig. 6. (a) Drive coil current (positive, in kA·turns), induced ring current (negative, in kA), and axial magnetic flux density for Sample $\mathrm{C}$ beyond penetration. (b) Scanning Hall probe measurements of Sample C.

values of $\approx 15 \mathrm{kA} / \mathrm{cm}^{2}$ and "static" values of $\approx 2.0 \mathrm{kA} / \mathrm{cm}^{2}$. The correlation disappears for higher values because the $0.3 \mathrm{~T}$ field used for sample cooling was not large enough to induce the maximum possible trapped field (the Bean critical state) for the samples with the two highest critical currents. For the other samples, the $J_{c}$ values measured by trapped-field methods are consistently $\approx 15 \%$ of the values measured under dynamic conditions. This is not surprising, because the measurement conditions are completely different. Transportbased critical current measurements are often conducted with an arbitrary electric field value, usually $1 \mu \mathrm{V} / \mathrm{cm}$, to define the location of the "critical" current on the $V-I$ (or $E-J$ ) curve of the sample. For many, but not all, sample geometries, this will put the YBCO well into the flux-flow regime, where slight increases in current will cause a huge jump in voltage so that the particular electric field criterion is not very significant [11]. In this case, the trapped-field measurement reflects the flux creep regime and an effective electric field criterion several orders of magnitude less than $1 \mu \mathrm{V} / \mathrm{cm}$. On the other hand, the maximum ring currents induced by pulsed fields are measured under conditions where we believe the effective 
electric field is in the range from 10 to $100 \mu \mathrm{V} / \mathrm{cm}$ and the relevant process is flux flow.

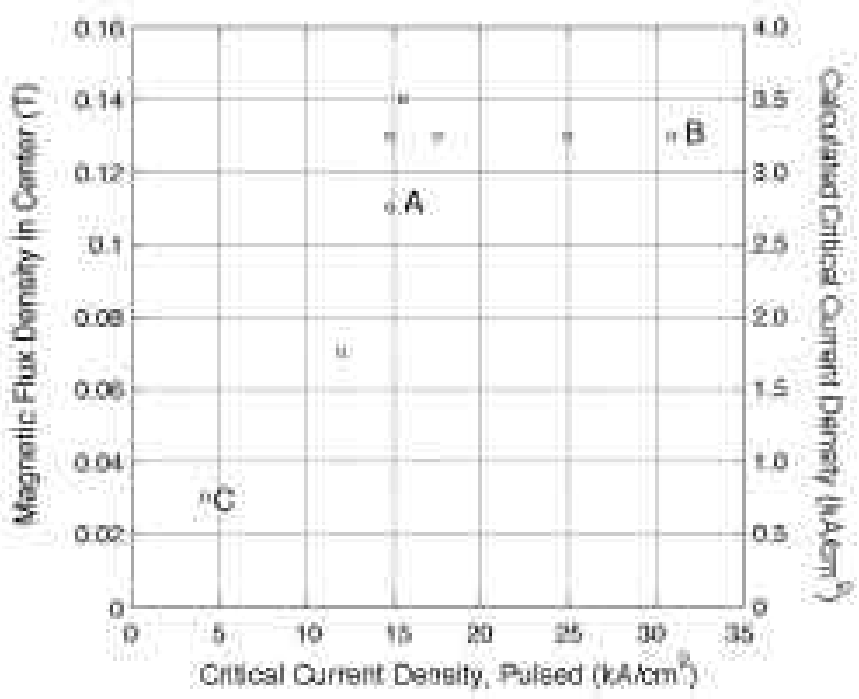

Fig. 7. Comparison of $J_{c}$ values at $77 \mathrm{~K}$ for 8 samples (Samples A, B, and C indicated) determined by measurements of the trapped magnetic flux density ( $y$ axes) and by response to pulsed magnetic fields ( $x$ axis).

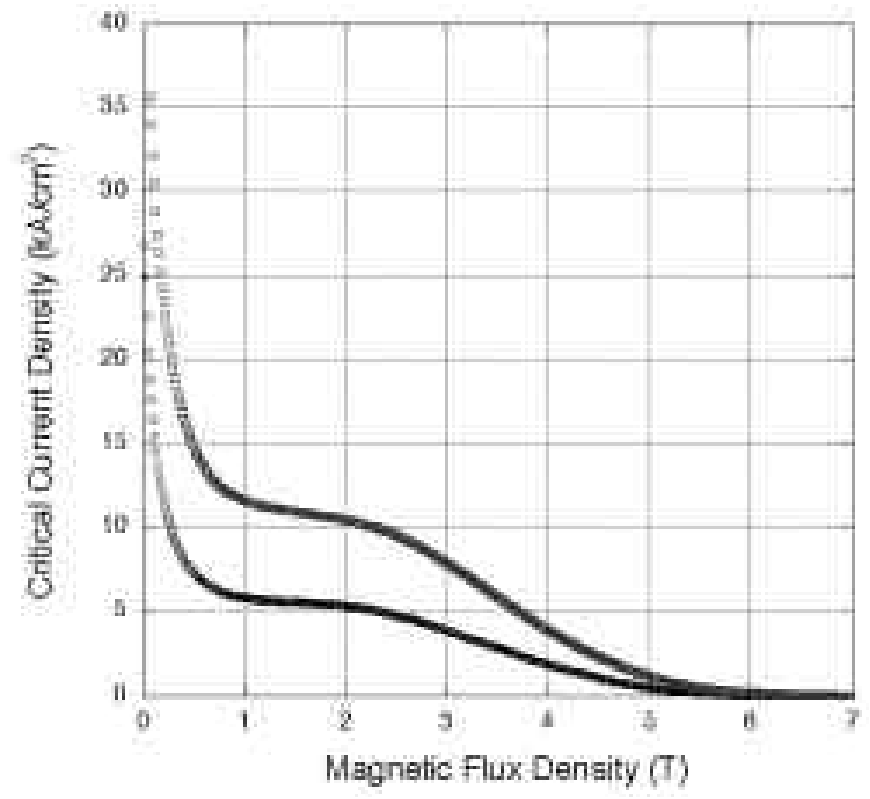

Fig. 8. Measurement of $J_{c}$ for Sample A in a region of strong pinning and a region of weaker pinning.

A third technique was also used to measure $J_{c}$, and the results are shown in Fig. 8. In this case, a 3-mm-dia. core drill was used to cut small cylinders out of the monolith for Sample A, with one small cylinder including a strong pinning region, and the other taken in a region with weaker pinning (see Fig. 1b). A vibrating sample magnetometer and magnetization loop analysis were employed according to methods discussed elsewhere [5]. Because the other two methods of $J_{c}$ measurement probe primary currents that circulate all the way around the ring, one expects the lower curve in Fig. 8 to agree most closely with the other methods, and this is the case. The relatively weak dependence of $J_{c}$ on applied magnetic fields below $2 \mathrm{~T}$ is another reason why the various measurement methods agree reasonably well. Although it is tempting to believe that the distribution of magnetic field and current in the samples is close to the Bean critical state in all three methods, the real situation is much more complicated, and the actual field distributions are likely different for each method. Because of the weak dependence of $J_{c}$ on $B$, this does not have a big effect on the results.

\section{COMPARISON TO OTHER SUPERCONDUCTING RINGS AND CONCLUSIONS}

Earlier work of this type has examined larger, multidomain melt-processed YBCO rings [3] and melt-cast BSCCO tube structures [8]. Three material systems have now been examined with shielding currents in the 10-40 kA range, and the results have been remarkably similar. The observed time constant for penetration in this case (3-4 ms) was shorter than the others, but this is probably due to the smaller size of the samples [12]. The high degree of uniformity among the samples in this study led to a more symmetric penetration process and more distinct and more easily observed changes in the magnetic field inside the ring. Although useful for increased understanding, increased symmetry in the penetration process seems to have little impact on device performance as long as the size of the shielding current remains usefully large. Designers of inductive FCLs can thus anticipate some flexibility in choice of superconducting material and sample dimension and uniformity.

\section{REFERENCES}

[1] D. A. Cardwell, "Processing and properties of large grain (RE)BCO," Mater. Sci. Eng. B, vol. 53, pp. 1-10, 1998.

[2] Y. S. Cha and T. R. Askew, "Transient characteristics of a high $T_{C}$ superconductor tube subjected to internal and external magnetic fields," IEEE Trans. Appl. Supercond., vol. 11, no. 1, p. 2485, 2001.

[3] T. R. Askew and Y. S. Cha, "Transient response of 50 kiloAmp Y-Ba$\mathrm{Cu}-\mathrm{O}$ rings and ring pairs to pulsed magnetic fields," IEEE Trans. Appl. Supercond., vol. 11 , no. 1, p. 3947, 2001.

[4] H. Zheng, H. Claus, L. Chen, A. P. Paulikas, B. W. Veal, B. Olsson, A. Koshelev, J. Hull, and G. W. Crabtree, "Transport currents measured in ring samples: Test of superconducting weld," Physica C, vol. $350 \mathrm{p}$. 17,2001

[5] H. Claus, U. Welp, H. Zheng, L. Chen, A. P. Paulikas, B. W. Veal, K. E. Gray, and G. W. Crabtree, "Critical current across grain boundaries in melt-textured YBCO rings," Phys. Rev. B, vol. 64, p. 144507, 2001.

[6] M. G. Ennis, T. J. Tobin, Y. S. Cha, and J. R. Hull, "Fault current limiter - Predominately resistive behavior of a BSCCO-Shielded-Core Reactor," IEEE Trans. Appl. Supercond., vol. 11, no. 1, p. 2050, 2001.

[7] N. Sakai, K. Ogasawara, K. Inoue, D. Ishihara, and M. Murakami, "Fabrication of melt-processed $\mathrm{RE}-\mathrm{Ba}-\mathrm{Cu}-\mathrm{O}$ bulk superconductors with high densities," IEEE Trans. Appl. Supercond., vol. 11, no. 1, p. $3509,2001$.

[8] Y. S. Cha and T. R. Askew, "Transient response of a high-temperature superconductor tube to pulsed magnetic fields," Physica C, vol. 302, p. $57,1988$.

[9] J. Ramboz, "Machinable Rogowski coil, design, and calibration," IEEE Trans. Instr. and Meas., vol. 45, no. 2, p. 511, 1996.

[10] L. Kojovic, "Rogowski coils suit relay protection and measurement," IEEE Computer Appl. in Power, vol. 10, no. 3, p. 47, 1997.

[11] J. E. Evetts, "Resistive transition and flux flow in superconducting materials," in Concise Encyclopedia of Magnetic and Superconducting Materials, Oxford: Pergamon Press, 1992, p. 478.

[12] Y. S. Cha, "Magnetic diffusion in high- $T_{c}$ superconductors," Physica $C$, vol. 330, p. 1, 2000 